COMENTARY

\section{Biological Clocks and Tenure Timetables: Restructuring the Academic Timeline}

\author{
Carol B. de Wet, Department of Geosciences, \\ Franklin \& Marshall College, Lancaster, Pennsylvania 17604, \\ c_dewet@email.fandm.edu
}

Gail M. Ashley, Department of Ceological Sciences, Rutgers, The State University of New Jersey, Piscataway, New Jersey 08854-8066,gmashley@rci.rutgers.edu

Daniel P. Kegel, OB-GYN Associates, Columbia Avenue, Lancaster, Pennsylvania 17603

The objectives of this paper are to (1) reaffirm the need for gender equity; (2) examine contributing factors to the attrition of women faculty; and (3) suggest alternative strategies to promote the full participation of women in geoscience higher education. Sections of the complete Commentary are abstracted below; the full text is posted at www.geosociety.org/pubs/gsatoday/.

\begin{abstract}
Despite decades of progressive social change by an active women's movement, federal and state legislation, and adoption of academic affirmative action policies, women geoscientists have not reached a critical mass in higher education. Women comprise only $12.5 \%$ of geoscience faculty in U.S. colleges and universities and only $10 \%$ at Ph.D. granting institutions. Senior women faculty tend to be marginalized from the academic power structure. A combination of biological factors, lifestyle choices, dual career pressures, double standards for social and professional interactions, and gender-based discrimination creates an effective filter, reducing women in geoscience departments to a surprisingly low level. There are two rungs on the ladder where women proportionally leave the discipline at a higher rate than men. One is continuing on to obtain a Ph.D.; the other is prior to, or at tenure. The present time frame for achieving tenure and promotion was established by men, for men, decades ago. Such a time frame is incompatible with women's biologic reproductive constraints, and as such, puts an unequal level of pressure and stress on women relative to their male professional counterparts. Only a significant change in the culture of science, and its traditional pathways, will create a geoscience community that has a sound base of gender equity. Strong leadership from innovative and far-sighted administrators and colleagues is required to introduce and foster institutional change that will reduce the conditions that leave women disadvantaged.
\end{abstract}

\section{Biological Factors-Childbearing Years and Tenure Trajectory}

Due to the inevitable tick of the biological clock, there is an unavoidable collision between a woman's optimum childbearing years and her career trajectory. Although career and family issues affect both men and women, there are issues unique to women who are starting a family.
The most fundamental gender specific issue is childbearing. Women face a difficult choice: wait to have children until their professional life is secure, but risk serious health consequences for their children (or selves), or bear their children earlier, and risk their professional success. Maternal mortality rates are four times higher among women 35 to 39 years old than those of ages 20 to 24, and babies of older women have higher mortality rates as well. In women age 35 or older, there is a four-fold increase in serious fetal complications, including stillbirth. This kind of emotional dilemma is what may lead some women leave the discipline. Those who stay in the profession experience tension that may seriously impact their quality of life, their career (research productivity, field and lab work), and their ability to successfully compete for jobs and grants. The overlap in biological and professional imperatives lasts for only a minor portion of a women's life, perhaps only 6 years out of a 35 -year career.

The risk of giving birth to a child with Down syndrome at age 35 is 1:270. By age 40 this risk is 1:106. Lethal chromosomal abnormalities are also more common with advanced maternal age, and risk of miscarriage is a significant concern. Only $10 \%$ of woman under the age of 20 experience spontaneous miscarriage. By age 40 this risk has more than doubled, to almost 34\%. Aging eggs, not simply overall good health, also affect a woman's fertility, and the natural incidence of chronic illnesses that complicate pregnancy increases with maternal age.

\section{Conclusions}

Attrition from the geosciences is higher for women than men at two critical points: after the M.Sc. degree, and between assistant and associate professor. Due to the inevitable tick of the biological clock, there is an unavoidable collision between a woman's optimum childbearing years and her career trajectory. Assuming that gender equity in science makes sense in terms of resources, diversified types of study, and balance, then causes for the rate of women's attrition must be sought.

Biological realities should to be acknowledged if we are to attain a critical mass of women in the geosciences. Stopping the tenure clock, allowing part time work for given time periods, and encouraging split positions are policies that already exist in some institutions. More responsive, flexible schemes for integrating work and family are essential to ensure women's full participation in higher education. At one time, it was considered impossible for female students to go on field excursions because of a lack of facilities. This "impossible situation" has been overcome, and there is no reason to doubt that the issues we have described can also be overcome.

Only by the retention and advancement of women can critical mass be achieved, after which women can begin to fill positions of power and influence. Women then can serve as role models for the next generation of scientists, encouraging more of them to enter and stay in science. This pool will then form the teaching basis for both academia and industry, and will potentially lead to a generation of managers and department heads who will be part of the ongoing transformation of the sciences. Only strong leadership today, by both administrators and faculty, can change the academic culture of priorities, workloads, reward structure and values to more closely reflect all of its constituencies and begin such a transformation.

Read the full text at www.geosociety.org/pubs/gsatoday/. 